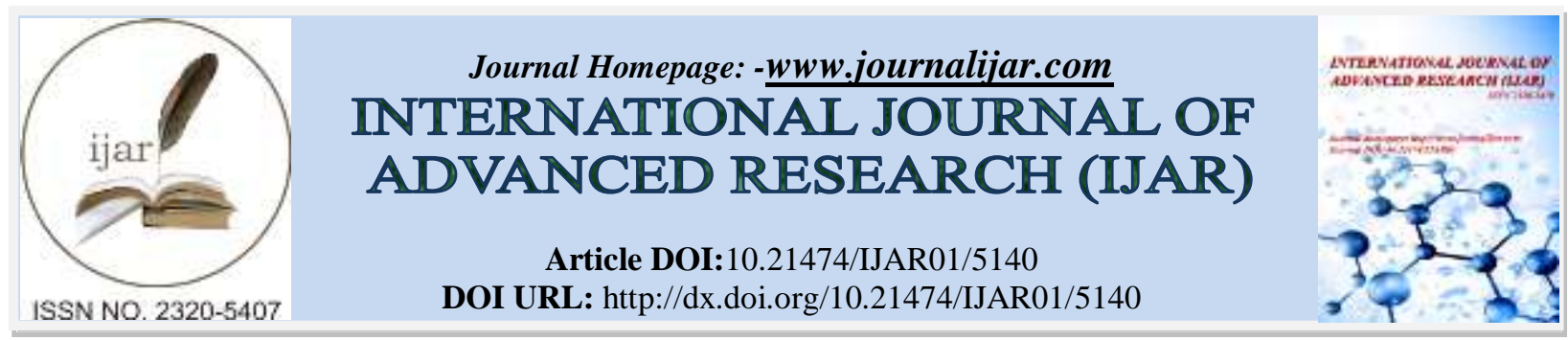

RESEARCH ARTICLE

\title{
ASSESSMENT OF CONTRIBUTING FACTORS IN EARLY CHILDHOOD CARIES AMONG RURAL POPULATION OF SULLIA TALUK.
}

Dr. Savitha Sathyaprasad, Dr. Krishnamoorthy S H, Dr. Musaffar T, Dr. Rekha R and Dr. Jerry George.

\section{Manuscript Info}

(..........................

Manuscript History

Received: 11 June 2017

Final Accepted: 13 July 2017

Published: August 2017

Key words:-

ECC, Demographic data, Feeding history, Dietary habits and Oral hygiene practices.

\section{Abstract}

Early childhood caries is a chronic, transmissible infectious disease, the most common, affecting primary teeth. It is the most prevalent (54\%) disease of childhood in India, a serious socio-behavioral and dental problem that afflicts infants and toddlers Prevalence of early childhood caries varies greatly in different countries.

Aim: To correlate the severity of early childhood caries to the etiological factors through a questionnaire study.

Methods: The sample size consisted of a total 150 healthy children aged from 0-6 years who reported to the department of pediatric dentistry KVG dental college and hospital in Sullia. A structured questionnaire was used to obtain information regarding demographic data, feeding history, dietary habits and oral hygiene practices. The status of dental caries was recorded using caries index and oral hygiene was recorded using oral hygiene index.

Results: 150 children with a mean age of 4.1 years were included in the study,39\% children were still bottle feed on demand and 52\% of children consumed more than once/day. $68 \%$ of children had poor oral hygiene and most of them brushes once daily with compromised parental supervision and most of the parents are uneducated. The mean $\mathrm{dmft}$ score was 4.3.Chisquare test was used to find the association between oral hygiene index with etiological factors .Percentage were used to analyze etiological contributing factors.

Conclusion: The high level of decay mainly attributed to poor oral hygiene, the decay also attributed to high sweet consumption, lack of regular dental visits and low socioeconomic status.

Copy Right, IJAR, 2017,. All rights reserved.

\section{Introduction:-}

Early childhood caries is a chronic, transmissible infectious disease, the most common, affecting primary teeth ${ }^{[1]}$. It is the most prevalent (54\%) disease of childhood in India, a serious socio-behavioral and dental problem that afflicts infants and toddlers ${ }^{[2]}$ Prevalence of early childhood caries varies greatly in different countries and it is a combination of frequent consumption of fermentable carbohydrates such as drinks especially when baby is sleeping with on demand breast or bottle feeding, oral colonization of cariogenic bacteria, poor oral hygiene and poor parenting.

Early childhood caries is considered as a serious public health problem as it is a complex disease of primary dentition which relay serious socio-behavioral issues that afflict mainly the infants and toddlers ${ }^{[3,4]}$ ECC, if left 
untreated, leads to deleterious effects such as pain, compromised chewing ability, malocclusion, phonetic problems, sub-optimal health, lower self-esteem. Hence, ECC is a social, political, behavioral, medical, psychological, economical, and dental problem that affects the quality-of-life. ${ }^{[5,6]}$ Dental problems impact considerably on selfesteem and quality of life and expensive to treat. ${ }^{[7]}$

The Objectives were;

To correlate the severity of early childhood caries to feeding history by questionnaire study.

To correlate the severity of early childhood caries to diet history by questionnaire study.

To assess the severity of early childhood caries to oral hygiene practices by questionnaire study and oral hygiene index.

\section{Methods:-}

The sample size consisted of a total 150 healthy children aged from0-6 years who reported to the department of pediatric dentistry KVG dental college and hospital in Sullia. A structured questionnaire was used to obtain information regarding demographic data, feeding history, dietary habits and oral hygiene practices. The status of dental caries was recorded using caries index and oral hygiene was recorded using oral hygiene index.

\section{Stastical analysis:-}

Chi-square test was used to find the association between oral hygiene index with etiological factors .Percentage were used to analyze etiological contributing factors. There was a significant association found between habit of sleeping while suckling the milk and oral hygiene status $(\mathrm{p}<0.005)$

\section{Results:-}

One hundred -fifty children met the inclusion criteria (73 males and 77 females). The age of the children ranged between 2 and 6 years with mean age of $4.47 .76 \%$ (114) were breast feeding and 24\% (36) were bottle feeding $.68 \%$ of peoples are unaware of ill effects of prolonged feeding.

Out of 80 children who has poor oral hygiene index $67.5 \%$ had the habit of sleeping while suckling the milk. There was a significant association found between habit of sleeping while suckling the milk and oral hygiene status $(\mathrm{p}<0.005)$,

\begin{tabular}{|c|l|l|l|l|l|}
\hline \multicolumn{2}{|l|}{ OHI } & Total & \\
\hline Habit of sleeping while sucking milk & good & Fair & poor & \\
\hline & yes & 4 & 33 & 54 & 91 \\
\hline & & $100.0 \%$ & $50.0 \%$ & $67.5 \%$ & $60.7 \%$ \\
\hline & no & 0 & 33 & 26 & 59 \\
\hline \\
\hline
\end{tabular}



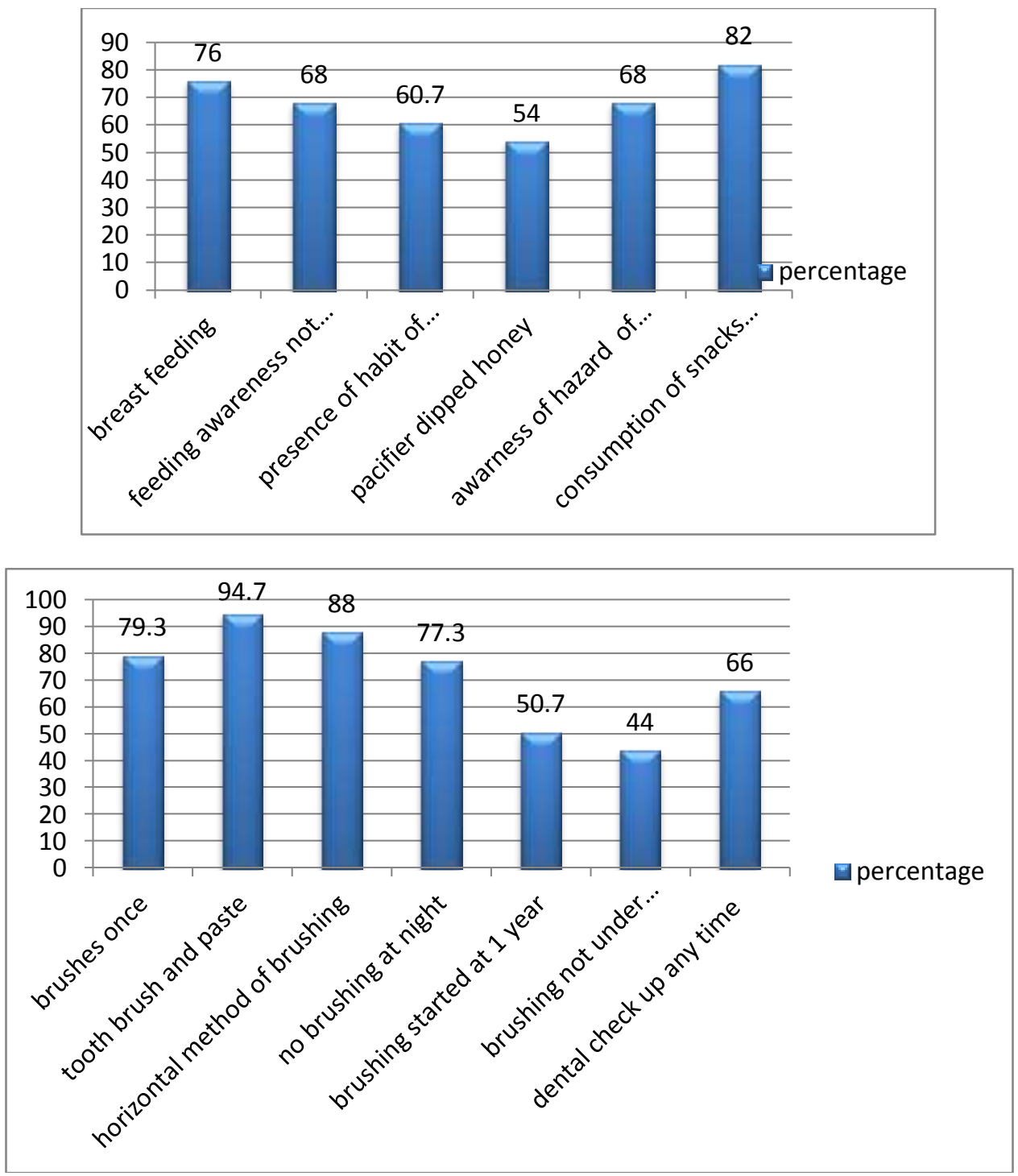

A high proportion of children brushes once daily (79.3\%) with only $22.7 \%$ brushing at night and most of them are using horizontal method of brushing (88\%). Most of children have started brushing at 1 year of age $(50.7 \%)$ and $45.3 \%$ started at $2^{\text {nd }}$ year and only $4 \%$ at 6 months. More than half of the Children brushes under supervision of parent (56\%).

A high proportion of children (66\%) not maintaining regular dental check-up. The level of dental decay is high, the mean caries index score is $4.08+-2.181$.

\section{Discussion:-}

In this study the results of oral examination revealed that poor oral hygiene is the main risk factor for decay.

The structured questionnaire showed that most of them uses tooth brush and paste (94\%) but $79.3 \%$ brushes once daily with $77.9 \%$ not brushes at night. Most of them have started brushing at age $1(50.7 \%)$.

According to AAPD, oral hygiene measures implemented no later than the time of eruption of the first primary tooth and twice daily use of fluoridated tooth paste with soft tooth brush on appropriate size is recommended. 
A toothbrush prophylaxis is efficient in removing plaque in most young children. It is also non-threatening to young children and serves to demonstrate the proper technique of brushing to the caregiver. The examiner retracts the child's lips and cheeks and demonstrates brushing along the gingival margins. The spongy handle of an ageappropriate toothbrush can be used as a prop to open the child's mouth. ${ }^{[8]}$

Preschool children neither understand nor have the manual dexterity to maintain good oral hygiene. Parental assistance and guidance are essential to reduce the risk of developing caries. There is a need for prolonged parental participation in the cleaning of preschool children's teeth. Parents should assess and supervise their preschool age child tooth brushing (brushing under supervision in this study were 56\%).

A significant correlation between frequency of sugar consumption and dental caries has been reported ${ }^{[9]}$. Children with ECC generally have a high frequency of sugar consumption, not only from fluids given in the nursing bottle, but also from sweetened solid foods. More than half (82\%) of the children in this study consumed sweets and snacks in between meals. This dietary characteristic cannot be ignored as being one of the most significant caries lesion risk factors in ECC. Sugars are most important cause of dental caries. Frequent consumption of carbohydrate containing snacks between meals is known to increase the amount of dental caries. Snacking several times and allowing snacks to stay on teeth cannot be neglected as an important cause of dental caries.

The possibility that there is some relation between the physical nature of food and the degree of dental-caries experience was noted. ${ }^{[10]}$

Among factors related to oral hygiene practices, two factors had a statistically significant relationship with ECC: The age of starting tooth brushing and the person responsible for child's oral hygiene. In the present study, children who started brushing at a later age had a higher prevalence of ECC. Most of children have started brushing at age 1 (50.7\%) and $45.3 \%$ started at 2 nd year and only $4 \%$ at 6 months. These finding are in agreement with the study conducted on Australian children ${ }^{[11]}$

Night time bottle feeding with juice, repeated use of a no spill cup and frequent consumption of sugar in between meals containing snacks or drinks increases the risk of caries.

The mean age of this study was 4.47 years. Two third had dental check-up at any time they required. $24.7 \%$ had first dental visit at age one.

The AAPD has recommend an initial dental consultation visit no later than 1 year of age to educate parents and provide anticipating guidance for prevention of dental diseases.

Infants and toddlers should not be expected to be cooperative during an oral examination. Crying and movements are developmentally age-appropriate behaviours for young children. Explaining expected behaviours to parents prior to, during and after infant care visits can help allay any fears and concerns they may have ${ }^{[8]}$

There is a simple six-step protocol for an infant oral care visit:

Caries risk assessment;

1. Proper positioning of the child (knee-to-knee exam);

2. Age-appropriate tooth brushing prophylaxis;

3. Clinical examination of the child's oral cavity and dentition;

4. Fluoride varnish treatment; and,

5. Assignment of risk, anticipatory guidance, self-management goals and counselling.

Risk-based caries prevention and management are important concepts in the prevention of ECC ${ }^{[12]}$. Parents should be encouraged to have infants drink from a cup as they approach their first birthday. Infants should be weaned from the bottle between 12 to 18 months of age. Parents play an essential role in developing a child's dietary behaviours and infancy is an important time to start healthy dietary habits ${ }^{[13]}$. In studies where oral health education was provided to mothers aiming to change attitudes and practices which can help reduce risks of poor infant oral health outcomes, it was found that some, but not all, risk behaviours were altered; despite knowledge of some of the risk factors, risk behaviours often continued. It is important that, if oral health promotion programs are to be implemented, to first identify why some health behaviours were adopted and others were not ${ }^{[14]}$ 


\section{Conclusion:-}

The severity of dental caries were high. The high level of decay attributed to poor oral hygiene practices (frequency and methods of brushing) lack of regular dental check-up, high level of sweet consumption. There is a great need for awareness which can be given to the parents as well as teachers about the risk factors to prevent further incidence \& progression of ECC in children and prospective long term studies and community based preventive programs to solve the continuing problem of early childhood caries.

\section{References:-}

1. Kowash MB. Severity of early childhood caries in preschool children attending Al-Ain Dental Centre, United Arab Emirates. European Archives of Paediatric Dentistry. 2015 Aug 1;16(4):319-24.

2. Masumo R, Bardsen A, Mashoto K, Astrom AN. Prevalence and socio-behavioral influence of early childhood caries, ECC, and feeding habits among 6-36 months old children in Uganda and Tanzania. BMC Oral Health. 2012 Jul 26;12(1):24.

3. Postma TC, Ayo-Yusuf OA, van Wyk PJ. Socio-demographic correlates of early childhood caries prevalence and severity in a developing country - South Africa. Int Dent J 2008;58:91-7.

4. Jose B, King NM. Early childhood caries lesions in preschool children in Kerala, India. Pediatr Dent 2003;25:594-600.

5. Kagihara LE, Niederhauser VP, Stark M. Assessment,management, and prevention of early childhood caries. J Am Acad Nurse Pract 2009;21:1-10.

6. Casamassimo PS, Thikkurissy S, Edelstein BL, Maiorini E. Beyond the dmft: The human and economic cost of early childhood caries.J Am Dent Assoc 2009;140:650-7.

7. Moynihan P, Petersen PE. Diet, nutrition and the prevention of dental diseases. Public health nutrition. 2004 Feb;7(1a):201-26.

8. Ramos-Gomez FJ, Crystal YO, Ng MW, Crall JJ, Featherstone JD. Pediatric dental care: prevention and management protocols based on caries risk assessment. Journal of the California Dental Association. 2010 Oct;38(10):746.

9. Hinds K, Gregory T. National diet and nutrition survey: children aged 1.5 to 4.5 years. Report of the dental survey Volume 2. HMSO: London; 1995

10. Prakash P, Subramaniam P, Durgesh BH, Konde S. Prevalence of early childhood caries and associated risk factors in preschool children of urban Bangalore, India: A cross-sectional study. European journal of dentistry. 2012 Apr;6(2):141

11. Hallett KB, O'Rourke PK. Social and behavioural determinants of early childhood caries. Aust Dent J 2003; 48(1):27-33.

12. Ng MW, Chase I. Early childhood caries: risk-based disease prevention and management. Dental Clinics of North America. 2013 Jan 31;57(1):1-6

13. Park S, Lin M, Onufrak S, Li R. Association of sugar-sweetened beverage intake during infancy with dental caries in 6-year-olds. Clinical nutrition research. 2015 Jan 1;4(1):

14. Retnakumari N, Cyriac G. Childhood caries as influenced by maternal and child characteristics in pre-school children of Kerala-an epidemiological study Contemporary clinical dentistry. 2012 Jan;3(1):2. 